\title{
Experiments with a novel CCD stellar polarimeter
}

\author{
D. Clarke $^{1}$ and D. Neumayer ${ }^{2}$
}

\author{
1 University Observatory, Acre Road, Glasgow, G20 0TL Scotland, UK \\ 2 Dept. of Physics and Astronomy, University of Heidelberg, Germany \\ e-mail: dirk.neumayer@urz.uni-heidelberg.de
}

Received 19 July 2001 / Accepted 30 November 2001

\begin{abstract}
Experiments and observations have been undertaken with "bread-board" equipment to explore the potential of a "ring" stellar polarimeter with a CCD camera, rather than photographic plates used in Treanor's (1968) original instrument. By spreading the polarimetric signal over a large number of pixels on the detector, design prediction suggests that the polarimetric accuracy could be $\sim \Delta p \pm 0.00001$ or $\pm 0.001 \%$ per frame or even better. Although the photon accumulations suggest that this was achieved, instabilities in the employed crude modulator system provided frame to frame measurements with a greater than expected scatter. Software was developed to reduce the data in a simple way. With a design using more professional components and perhaps with more sophisticated reduction procedures, the full potential of the method should be achievable with the prospect of high precision polarimetry of the brighter stars. As an experimental bonus, the employed CCD chip was found to be free from any measurable polarizational sensitivity.
\end{abstract}

Key words. instrumentation - polarimeters: techniques - polarimetric

\section{Introduction}

Polarimetry of extended sources is now regularly undertaken using CCD detectors following an appropriate modulator. For example, Scarrott (1991) and his group have produced polarization vector maps of bi-polar nebulae and galaxies. The 2D properties of CCDs can also be utilised to perform spectropolarimetry, the modulator being located just prior to the entrance slit of a spectrometer. Results from such exercises have important bearing on stellar atmospheric processes (see, for example, Schmid \& Schild 1994). Broad band (filter) stellar polarimetry based on point-like, focused images has also been undertaken by Magalhães et al. (1996), for example, with accuracies for the degree of linear polarization, $p$, quoted as $\Delta p \sim \pm 0.03 \%$ or \pm 0.0003 , the uncertainties being marginally larger than those predicted by the noise on the collected number of photons.

Stellar polarimeters employing photomultiplier detectors are commonly claimed to achieve measurement accuracies matching those predicted by photon counting statistics. For instruments determining the Stokes parameters, $Q$ and $U$, or, more often, normalised Stokes parameters (NSPs), expressed as $q=Q / I$ and $u=U / I$, where $I$ is

Send offprint requests to: D. Clarke, e-mail: d.clarke@astro.gla.ac.uk the beam intensity, the simplest conceptual measurements involve the determination of appropriate orthogonally resolved components, allowing noise estimates to be readily calculated.

Consider just one parameter, say $q$, such that

$q=\frac{Q}{I}=\frac{N_{q \perp}-N_{q \|}}{N_{q \perp}+N_{q \|}}$

where the values $N_{q \perp}$ and $N_{q \|}$ correspond to photon counts associated with the perpendicular and parallel resolved components. For low levels of polarization, $N_{q \perp} \sim$ $N_{q \|}=N_{q}$ and simple error analysis provides an uncertainty estimate of $\sigma_{q}= \pm \sqrt{2 / N_{q}}$. Thus, in order to achieve an accuracy such that the uncertainty in $q \sim$ \pm 0.0001 , a total photon count $\sim \sqrt{2} \times 10^{8}$ is required. One of the problems with photon counting photometry is that of "dead-time", this corresponding to the time interval required between the production of two photoelectrons for each to be counted as separate events. Pulse overlap leads to counting losses and photometric non-linearity. The problem also affects photon counting polarimetry and depending on whether corrections are made, variable systematic errors affect the compared data. Clarke \& Naghizadeh-Khouei (1994) have drawn attention to such a problem in observational studies related to the detection of temporal variations of interstellar polarization. To 
avoid dead-time effects, it is necessary to limit the photon count rate to be no greater than $\sim 5 \times 10^{5} \mathrm{~s}^{-1}$. For bright stars, this is sometimes done by the introduction of neutral density filters within the instrument, at the expense of using longer integration times to achieve the necessary large photon count, so performing the exercise with inefficient use of the telescope collection aperture.

As an example of the integration time required with such controlled maximum count rates, consider the situation where an accuracy of $\sigma_{q} \sim \pm 0.0001$ is required. A typical integration time would therefore be $\left(\sqrt{2} \times 10^{8}\right) /(5 \times$ $\left.10^{5}\right) \approx 300 \mathrm{~s}$. Such an inefficient photon collection procedure is a large penalty to pay when the original collected flux might have carried a photon count rate as high as $10^{8} \mathrm{~s}^{-1}$, i.e., the polarimetric information to the required accuracy is available in a time window of a couple of seconds.

Although CCD devices do not suffer from the deadtime problem, they have a limitation in that their individual pixels have a "full well capacity" with the photon detection accumulations having a maximum value above which over-exposure occurs. For a typical CCD, this will be $\sim 10^{5}$ electrons per pixel, so limiting the accuracy of a determined NSP to about \pm 0.003 per pixel. When CCD detectors are used for photometry, stellar images are spread over many pixels allowing more photons to be detected per frame, with a commensurate improved accuracy. Based on the immediate values above, if 100 pixels were to be used with uniform exposure close to their full well capacity, the polarimetric accuracy would be improved by $\sim \sqrt{100}=10$, i.e., $\sigma_{q}$ now becoming $\sim \pm 0.0003$. If $10^{5}$ pixels were to be used, the potential accuracy is $\sim \pm 0.00001$; for a chip offering $10^{6}$ pixels, all devoted to a single polarimetric determination, the potential accuracy is $\sim \pm 0.000003$. Thus, if the stellar source is bright, rather than reducing the photon detection rate by using neutral filters, it makes sense to use a CCD detector and spread the light over the pixel structure in some kind of controlled way.

One such experiment for measuring individual stars was by Clarke \& Naghizadeh-Khouei (1997) whereby the brightness values were obtained from orthogonally resolved images of the telescope aperture set side by side on the CCD chip. By taking alternate frames, after flipping the orthogonally resolved components by the use of a half-wave plate, the system removed effects of any variable atmospheric extinction during the data accumulation and was self-calibrating against effects of non-uniformity of the pixel sensitivities, so avoiding the usual flat-fielding exercises. Some 3000 pixels were used and accuracies of $\sigma_{q} \sim \pm 0.0001$ were readily achievable. In addition, it was also possible to investigate the instrumental polarization imposed by variation of the reflection properties over the telescope mirror surfaces.

Another possible approach is an adaptation of the modulator of Treanor (1968), originally applied to star fields and using photographic plates as the recording medium. In this design the polarimetric information is obtained by rotating a polarizer and a tilted glass plate just prior to the telescope focus where the detector is placed. The modulator causes any stellar point image to execute a circle with the record of intensity around the annulus expressed by

$$
S(\alpha)=\frac{1}{2} G(\alpha)[I+Q \cos 2 \alpha+U \sin 2 \alpha]
$$

with the fiduciary angle of the modulation $\left(\alpha=0^{\circ}\right)$ corresponding to positive $Q$ of the Stokes parameter reference frame. The scaling factor $G$ relates the pixel response to unit intensity and with its $\alpha$ dependence represents the fact that each pixel carries an individual sensitivity. Flatfielding procedures are therefore required to abstract the true polarimetric information. By examining the corrected $S(\alpha)$ variation, $q$ and $u$ can be determined, so providing a value for $p$ and the direction of vibration, $\beta$, relative to the reference frame, given by

$$
p=\left(q^{2}+u^{2}\right)^{1 / 2} \quad \text { and } \quad \tan 2 \beta=u / q
$$

Because of the appearance of the raw data image, this system may be conveniently referred to as a "ring" polarimeter. One of the attractions of the arrangement is that all the polarimetric information is contained in a single frame and that any variations in atmospheric transparency are smoothed out during the exposure.

A prototype instrument based on Treanor's design, but using a CCD detector, has been built and tested in the laboratory and on stellar sources. The spreading out of the radiation in the form of an annulus over a large number of pixels is attractive in terms of the potential of high polarimetric accuracy. Because of the requirement to determine the brightness variation around a record with circular symmetrical structure imposed on a matrix comprising rectangular or square pixels, special data reduction algorithms were developed. As well as testing out the basic polarimetric design and potential, it is obviously important to evaluate the data reduction procedures. In the following sections, the basic instrument and computer algorithms are presented.

\section{The prototype instrument}

To provide a completely independent system, an instrument was constructed with its own small telescope $(75 \mathrm{~mm}$ objective, focal length $=350 \mathrm{~mm}$ ) capable of monitoring the brighter stars. A schematic of the arrangement is depicted in Fig. 1. Following the collection aperture, in optical path sequence, the instrument comprises a flip-mirror system to allow visual inspection of the field of view, a filter wheel to allow selection of the wavelength passband, the polarimetric modulator driven by an external motor, a transfer lens (50 mm photographic camera lens), an optional fixed polaroid (for use with an alternative waveplate modulator) and the CCD detector. It may be noted that for similar systems to be attachable to larger telescopes, the whole assembly could be set deeper in from the focal 


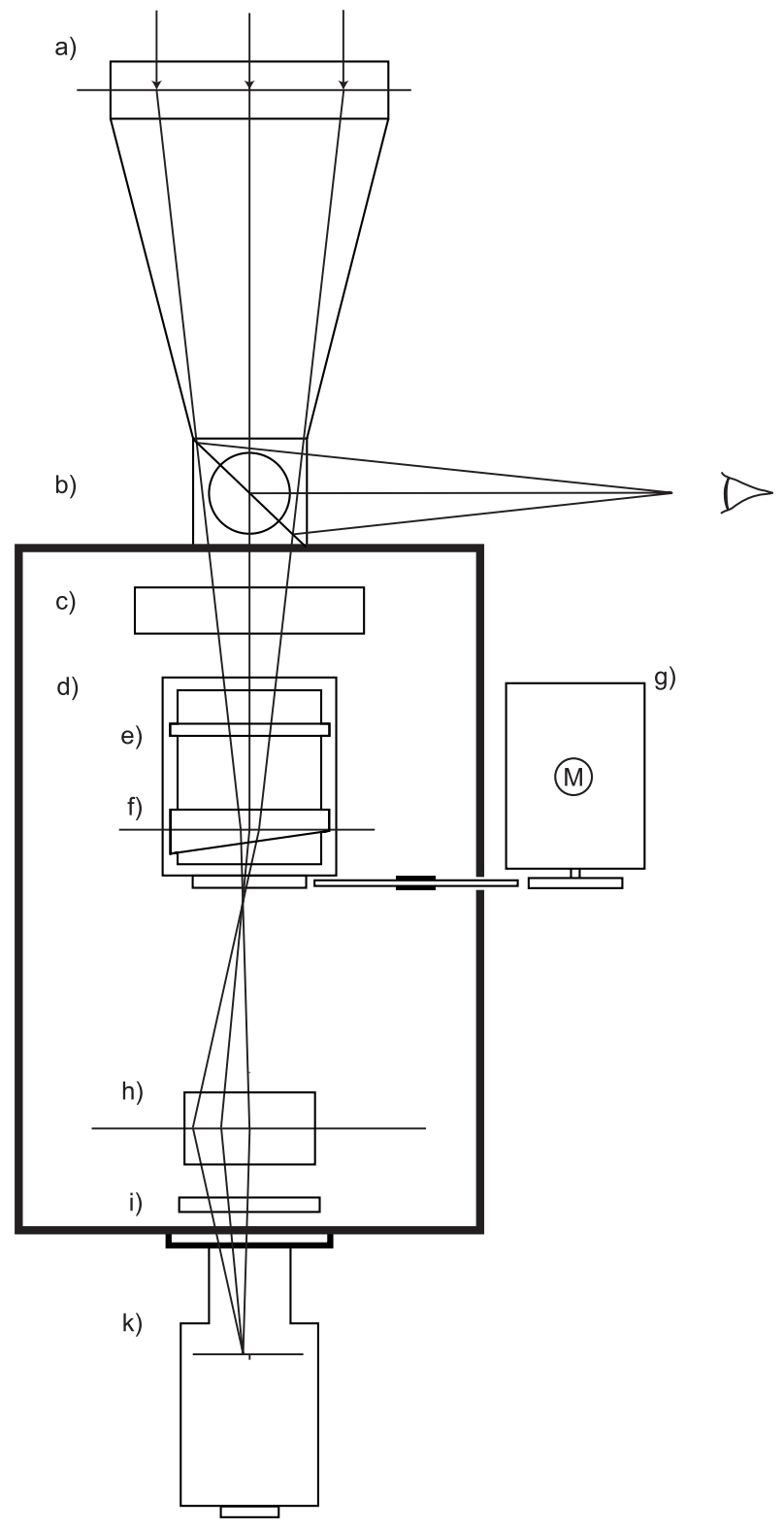

Fig. 1. A cross-sectional drawing of the prototype ring polarimeter shows a) the $75 \mathrm{~mm}$ objective; b) the flip-mirror field viewing system; c) the filter wheel passband selection; d) the polarimetric modulator - a metal tube held in a ball-race holding e) a sheet polarizer and f) a small angled glass wedge; g) a synchronous motor; h) a $50 \mathrm{~mm}$ image transfer lens; i) an optional sheet polarizer; k) CCD camera. Three light paths are overlaid to emphasize the purpose of the glass wedge to deviate the point image and the transfer lens to refocus the original telescope image.

plane and the CCD could be positioned there without the need for the image transfer optics.

Many polarimeters which use photomultipliers for signal detection employ a rotating half-wave plate (achromatic or superachromatic) prior to a fixed polarizer. For such a system, the modulation frequency, as described in Eq. (2), is simply doubled. The instrumental advantage here is that the polarized light falling on the detector has a fixed direction of vibration. This arrangement obviates

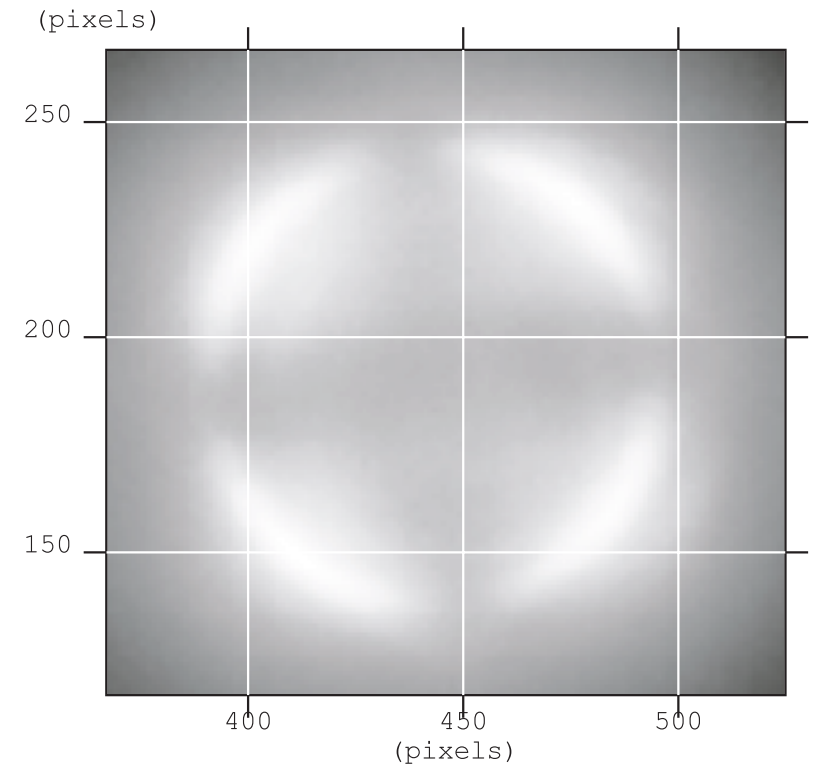

Fig. 2. The image of a laboratory artificial $100 \%$ polarized star obtained with the prototype polarimeter using a tilted rotating half-wave plate prior to a fixed polarizer. Note that the intensity around the image is modulated four times.

the generation of spurious signals from the detector should it possess any polarizational sensitivity. As the CCD's response to polarized radiation was uncertain, trials were conducted (see Fig. 2) using a rotating tilted half-wave retarder prior to a fixed polarizer. The design was not pursued, however, as the inclination of the plate to the light path causes it to behave as a partial polarizer prior to the fixed polarizer. On rotation of the plate, an additional modulation on the output signal is produced which is generally larger than that produced by any weak polarization in the radiation of the star itself.

The chosen modulator comprised a sheet polarizer and glass wedge. The latter devices are obtainable with a variety of wedge angles, the choice dictating the size of the annular record on the CCD. By choosing a suitably angled wedge, either a large star field (see Fig. 3) might be analysed on a single frame or, alternatively, a single bright star (see Fig. 4) can be analyzed with high accuracy. For the majority of experiments the wedge angle was $\sim 1.5$. The motor ran at $500 \mathrm{rev} / \mathrm{min}$ but via the gearing system, the modulator cell rotated at $\sim 715 \mathrm{rev} / \mathrm{min}$, sufficiently fast to average out intensity scintillation noise and effects of variable atmospheric transparency during the exposure. Also, this high speed eliminated any effects of there being a non-integer number of revolutions over the nominated exposure time. The CCD camera was a model HX516 from Starlight-Xpress using a Sony ICX084AL HyperHAD chip with pixel size $7.4 \times 7.4 \mu \mathrm{m}$ and a format of $660 \times 494$ pixels.

For the laboratory tests, allowing the proving out of the reduction software, the complete instrument was readily set up to record artificial stars (pin-holes). For the more complete exercise, the polarimeter box was mounted on a thick aluminium plate and attached to the side of the $0.5 \mathrm{~m}$ 


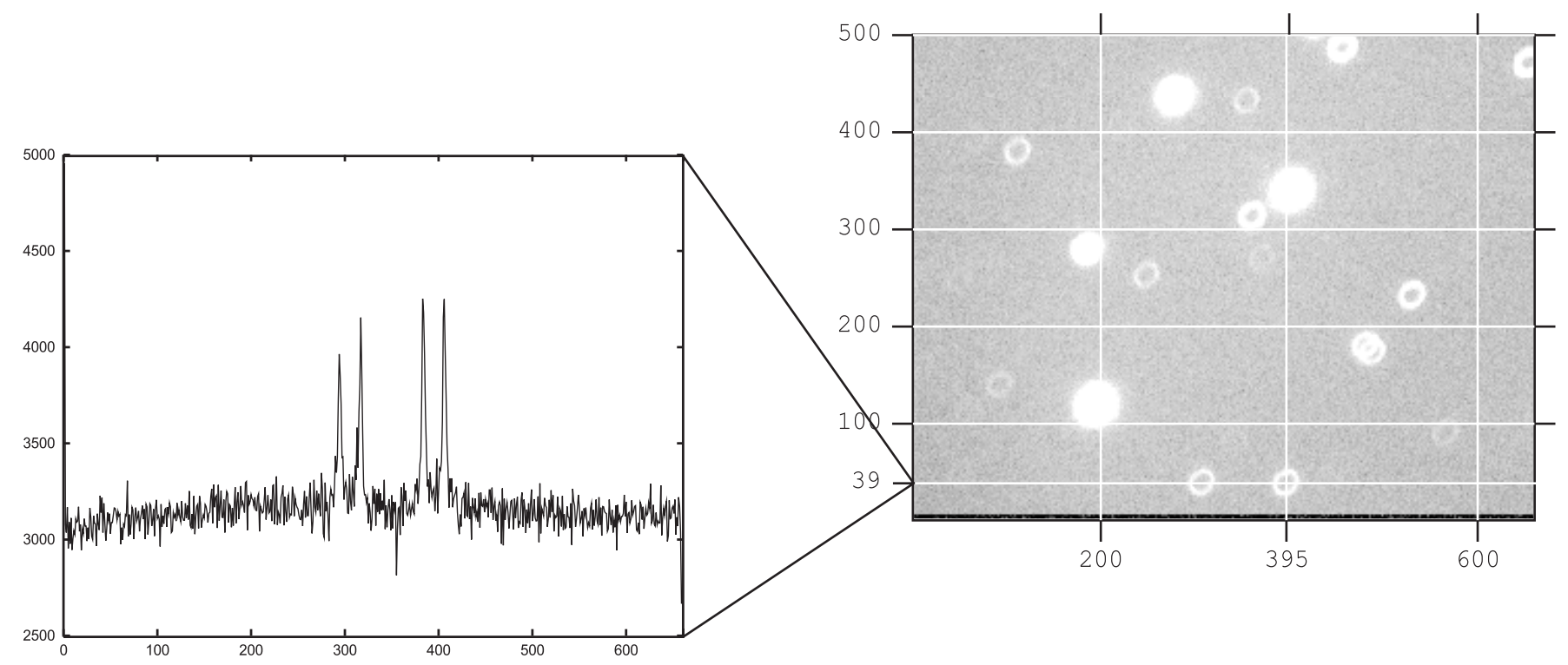

Fig. 3. The right-hand part is a CCD frame of part of the Pleiades cluster recorded by an early version of the polarimeter. These data were not fully analysed to determine polarizations as the images were elliptical resulting from motor induced vibrations. The left-hand part displays the signal levels (ADU) through pixel row number 39 running through two star images.

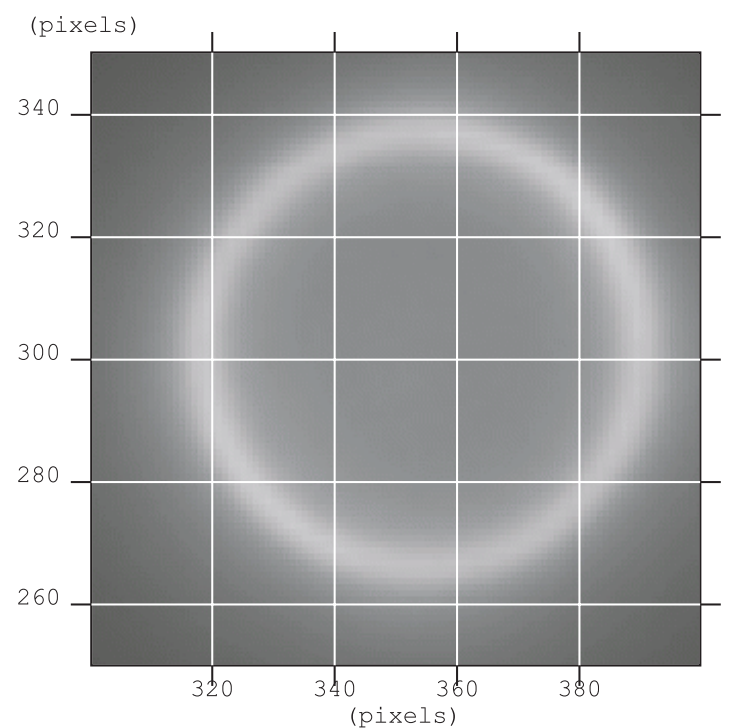

Fig. 4. A CCD frame for $\alpha$ Leonis showing the ring obtained from an essentially unpolarized star. In an attempt to obtain the highest polarimetric accuracy for an individual star, a wedge was chosen within the modulator to produce an image nearly filling the format of the chip.

telescope at the Cochno site at the University of Glasgow. The latter instrument was used simply as a platform to provide tracking and guiding. Additional connecting bars and damping materials were used to reduce the effects of vibration caused by the high speed motor.

\section{Data reduction}

The intensity signal produced by the polarimetric modulator comprises a mean level with a superimposed oscillatory component described by an amplitude and phase, these three unknowns being relatable to the parameters $I, Q$ and $U$. There are many possible strategies for retrieving the polarimetric information but the one selected involves three integrated intensities around sections of the annulus to allow direct determination of the NSPs. Assuming that the polarizer rotates $M$ times during the exposure, so producing $M$ overlaid circles, and following a flat-fielding exercise, the three signals may be written as

$$
\begin{aligned}
S_{1} & =M \int_{0}^{2 \pi} I(\alpha) \mathrm{d} \alpha=M \pi I \\
S_{2} & =M \int_{0}^{\frac{\pi}{2}} I(\alpha) \mathrm{d} \alpha+M \int_{\pi}^{\frac{3 \pi}{2}} I(\alpha) \mathrm{d} \alpha \\
& =2 M \int_{0}^{\frac{\pi}{2}} I(\alpha) \mathrm{d} \alpha=M\left(\frac{\pi}{2} I+U\right) \\
S_{3} & =M \int_{\frac{\pi}{4}}^{\frac{3 \pi}{4}} I(\alpha) \mathrm{d} \alpha+M \int_{\frac{5 \pi}{4}}^{\frac{7 \pi}{4}} I(\alpha) \mathrm{d} \alpha \\
& =2 M \int_{\frac{\pi}{4}}^{\frac{3 \pi}{4}} I(\alpha) \mathrm{d} \alpha=M\left(\frac{\pi}{2} I-Q\right) .
\end{aligned}
$$

The NSPs may then be derived as

$q=\frac{Q}{I}=\pi\left(\frac{1}{2}-\frac{S_{3}}{S_{1}}\right) ; \quad u=\frac{U}{I}=\pi\left(\frac{S_{2}}{S_{1}}-\frac{1}{2}\right)$.

One of the advantages of integrating with the above limits is that the reduction process itself acts as a filter by ignoring some of the potential spurious harmonics from the signal, concentrating only on those given by $2+4 n$, where $n=0,1,2,3$, etc.

In estimating the uncertainties associated with the NSP measurements, it will be appreciated that the values of $q$ and $u$ are obtained partially over the same portion of the modulation profile, so that the photo-electron noise 


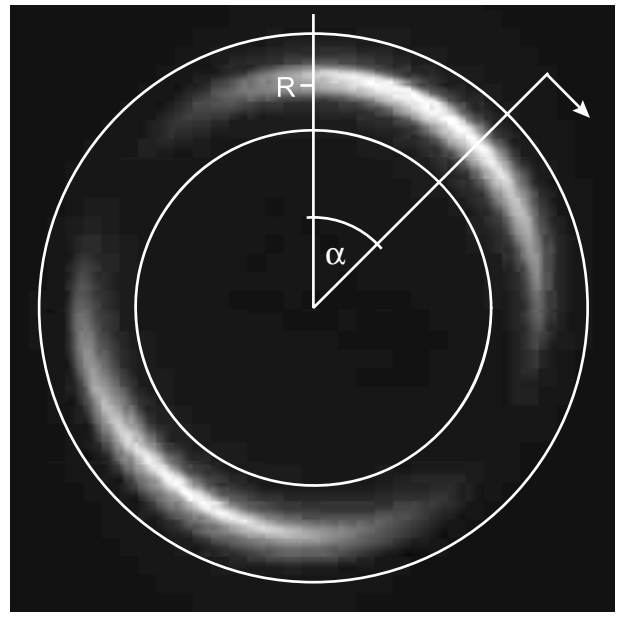

Fig. 5. A frame showing the record of the star $\alpha$ Leonis, the light made $100 \%$ polarized by placing a filter over the telescope aperture. The same modulator wedge was used as for the frame depicted in Fig. 4. The behaviour of the Malus Law is clearly seen. Imposed on the image are the bounds in radius, $R$, over which the contributing intensities are obtained over the range of $\alpha$ running from 0 to $2 \pi$.

introduces a correlation between the determined NSPs. Stewart (1985) has shown, however, that such correlations are insignificant if $p<10 \%$ and if the photo-electron count is greater than a few thousand. Hence, from an analysis based on uncorrelated Gaussian error propagation,

$\Delta q= \pm \frac{1}{\sqrt{G}} \sqrt{\left(\frac{-\pi}{S_{1}} \sqrt{S_{3}}\right)^{2}+\left(\frac{\pi S_{3}}{\left(S_{1}\right)^{2}} \sqrt{S_{1}}\right)^{2}}$

and

$\Delta u= \pm \frac{1}{\sqrt{G}} \sqrt{\left(\frac{\pi}{S_{1}} \sqrt{S_{2}}\right)^{2}+\left(\frac{-\pi S_{2}}{\left(S_{1}\right)^{2}} \sqrt{S_{1}}\right)^{2}}$

with

$\Delta p= \pm \frac{1}{p \sqrt{G}} \sqrt{q^{2}(\Delta q)^{2}+u^{2}(\Delta u)^{2}}$.

For cases of stars with zero or very small polarizations

$\Delta p= \pm \frac{\pi}{\sqrt{G}} \sqrt{\frac{3}{4 S_{1}}}= \pm \sqrt{\frac{3 \pi}{4 G M I}}$.

To increase the accuracy of the polarimetry, high photoelectron counts $(G M I)$ are necessary. Since the well capacity of the pixels is limited, it is a requisite that the image be spread over an area of the chip. For example, the ring image of an out of focus star providing an outer radius $R_{\text {out }}=100$ pixels and inner radius $R_{\text {out }}=80$ pixels involves about 22500 pixels. If now a frame records 20000 photo-electrons per pixel, the total photo-electron count is $\sim 4.5 \times 10^{8}$ and the uncertainty of $p$ is $\sim \pm 7 \times 10^{-5}$.

Since the determination of $p$ is obtained from the combination of signals from many pixels, it is important that the flat-fielding exercise is performed to good accuracy. Effects of fringing and vignetting must also be carefully

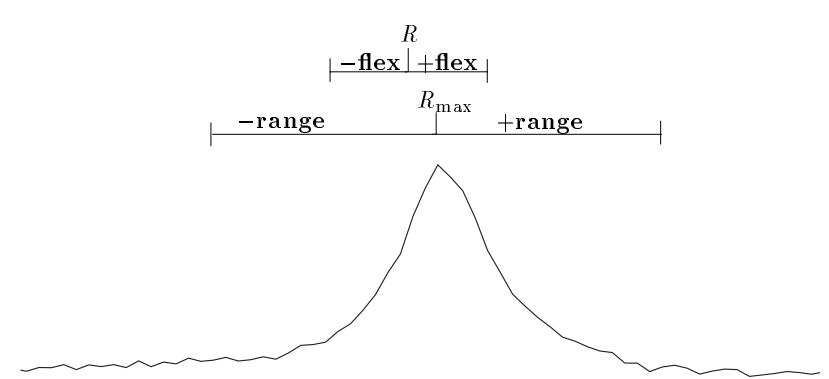

Fig. 6. The peaked curve displays the intensity behaviour along a radius from the center of a ring image, with the position of $R_{\max }$ and the zones of $R \pm$ flex, $R \pm$ range indicated.

investigated in the data reduction. In the observational procedures, repetitive flat-fielding needs to be performed to ensure that the noise introduced by pixel calibration is much smaller than the photon shot noise of any single polarimetric frame.

\section{Software development}

Due to the uniqueness of the recorded images, specially developed software was required to reduce the data. Following the dark signal removal and application of flatfielding corrections associated with general CCD frame procedures, the polarimetric reduction involved:

1. Identification of ring images and determination of the circle centers and radii;

2. Production of the intensity variation around the annulus of each circle;

3. Determination of the NSPs.

The ring centers are readily found essentially by first scanning the pixel rows and noting the column corresponding to the mid separation of any pair of intensity peaks. The same circle will be cut several times as the row number progresses and a mean value for the column containing the ring center is obtained. An identical procedure is then applied to an orthogonal scan of the pixel columns.

A ring around the center of the chosen circle with radius, $R$, is then considered. The inner radius of the ring is $R_{\text {in }}=R$ - range and the outer radius is $R_{\text {out }}=$ $R+$ range. The value for range is automatically determined by firstly finding the position $\left(R_{\max }, \alpha\right)$ on the annulus where $I_{\max }$ is found. Then starting from the ring center and exploring towards $\left(R_{\max }, \alpha\right)$ in a radial direction, the intensity profile is examined. It is expected that the values will rise from the background level to $I_{\max }$ and then fall back again (see Fig. 6 ). The two positions, $R_{1}$ and $R_{2}$, where the intensity profile crosses the value $0.1 \times I_{\max }$ are noted. This threshold intensity level is arbitrary but provides a realistic way of utilising the majority of the collected photons without introducing noise from pixels containing responses to low light levels. The mean of $\left|R-R_{1}\right|$ and $\left|R-R_{2}\right|$ gives the value for range. By dividing the ring into 8 sectors each of $45^{\circ}$, appropriate additions allow determinations of $S_{1,2,3}$. According to the sector labelling 

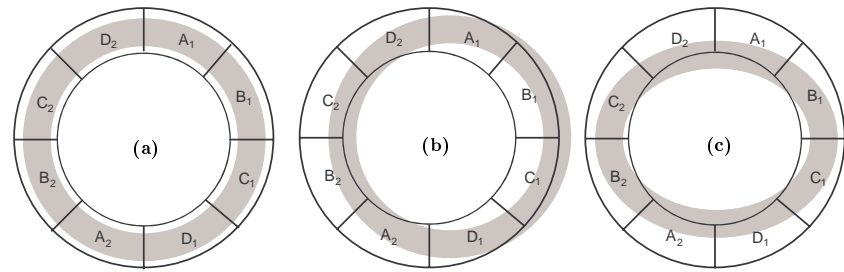

Fig. 7. The pixel area from which the polarimetry is undertaken is depicted as an annulus divided into 8 equal segments each covering $45^{\circ}$. In a), a perfect circular image produced by the modulator is correctly centered; b) conveys how an incorrectly determined center for the image will distort the signal as some of the illuminated pixels lie outside the zones from which the intensities are determined; in c), the sketch conveys how an elliptic record would produce a false $\sin (2 \alpha)$ modulation.

as in Fig. 7, the integrations corresponding to Eqs. (4)-(6) are given by the sums of the zone intensities as

$S_{1}=A_{1}+A_{2}+B_{1}+B_{2}+C_{1}+C_{2}+D_{1}+D_{2}$

$S_{2}=A_{1}+A_{2}+B_{1}+B_{2}$

$S_{3}=B_{1}+B_{2}+C_{1}+C_{2}$.

If the ring center is incorrectly determined, a spurious modulation signal would be generated (see Fig. 7b). However, in the conducted tests, the effect was negligible as the accuracy of determining the ring centers is estimated to be \pm 1 pixel. Compared to a ring radius of about 100 pixels, this represents a very small uncertainty; further, the pixels that might get lost as a result of the displacement are those at the borders of the ring where the photo-electron contribution to the determination is at its lowest. If the recorded image is not perfectly circular and the range is too small, a spurious $\sin (2 \alpha)$ signal is generated (see Fig. 7c). Both the imperfect centering and any image ellipticity are reduced by setting the range sufficiently wide.

The intensity variation around the annulus is readily derived by scanning through the pixels from the center of the ring along various radius vectors according to the angle, $\alpha$. As each section of the annulus is approached the recorded intensity behaviour is similar to that for the row and column scans described earlier in connection with the determination of the ring centers. The position of the maximum value, $R_{\max }(\alpha)$, is set within an interval, $R \pm$ flex. If the annular image were perfectly circular, values of $R_{\max }$ would be constant but, because of uncertainties in the determination of the ring center, or telescope tracking irregularities, or discretisation of the pixel matrix, this is almost never the case. Intensity values from the pixels in the interval $\left[R_{\max } \pm\right.$ plotrange $\left.(\alpha)\right]$ are summed and stored. For each $\alpha$, stepped by $1^{\circ}$, the radial summation is performed with a step size of 1 pixel, each identified by converting the polar co-ordinates $(R, \alpha)$ to their Cartesian counterparts $(x, y)$. For values of $\alpha=0^{\circ}, 90^{\circ}, 180^{\circ}$ and $270^{\circ}$, the converted co-ordinates correspond exactly to the rows and columns of the CCD chip. Since the detector matrix comprises square pixels, for intermediate values of

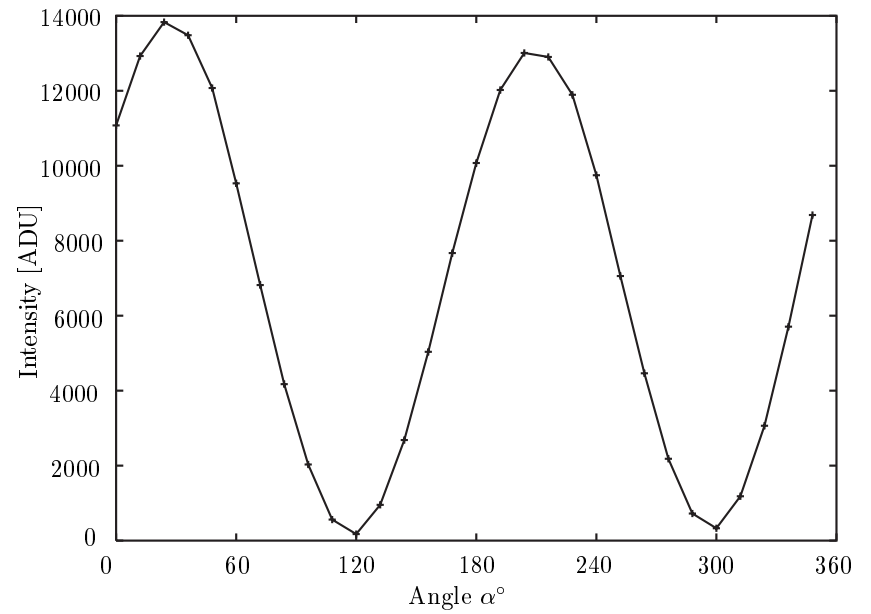

Fig. 8. An example of the intensity profile around the circular image of a fully polarized stellar source, as in Fig. 5, showing the expected behaviour of the Malus law $(I \propto \cos 2 \alpha)$. The data have been binned in $12^{\circ}$ sections around the annulus.

$\alpha$, the conversion provides a radius vector path which is not regular, i.e., the $x, y$ values are determined as floating point numbers and the appropriate pixel is identified by taking the integer parts. More sophisticated interpolation schemes could be imagined but, because of the smallness of the pixel element size relative to the seeing induced spread of the annular image along the radius vector, this simple method produced smooth $I(\alpha)$ variations. Values of $q, u$ are calculated by the appropriate binning of the $I(\alpha)$ values according to Eqs. (4)-(6) (or Eqs. (12)-(14)).

\section{Discussion of results and conclusion}

In an exercise to investigate the potential of the ring instrument as a high accuracy polarimeter, most of the observations concentrated on individual bright stars, the chief candidate being $\alpha$ Leo. Measurements by Behr (1959) indicate that this object essentially exhibits zero polarization. Unfortunately no bright stars were available with well defined $p(\lambda)$ values to act as measurement calibrators.

The efficiency of the system was checked by covering the small telescope aperture with a sheet polarizer and exposures were made with $120 \mathrm{~s}$ integration. A typical record is shown in Fig. 5, the strong modulation giving the appearance of there being two croissants placed horn-tohorn. After integrating the ensuing $I(\alpha)$ over $\alpha$ intervals of $12^{\circ}$, the resulting curve following a $\cos 2 \alpha$ variation as expected from the Malus law (see Fig. 8). From two such frames, the resulting value of $p$ was $98.3 \% \pm 0.1 \%$. The departure from $100 \%$ may be due either to the imperfect efficiency of the sheet polarizer, particularly at the red end of the embraced spectral range, or may originate through imprecise background subtraction - the observations were made on a night of strong auroral activity.

A recorded frame of $\alpha$ Leo, essentially providing unpolarized light is shown in Fig. 4 where it can be seen that the ring image is essentially uniform. From 10 such exposures, the mean polarimetric values were: $q=0.0090 \pm$ $0.0015, u=0.0024 \pm 0.0016$ with $p=0.0094 \pm 0.0016$, 


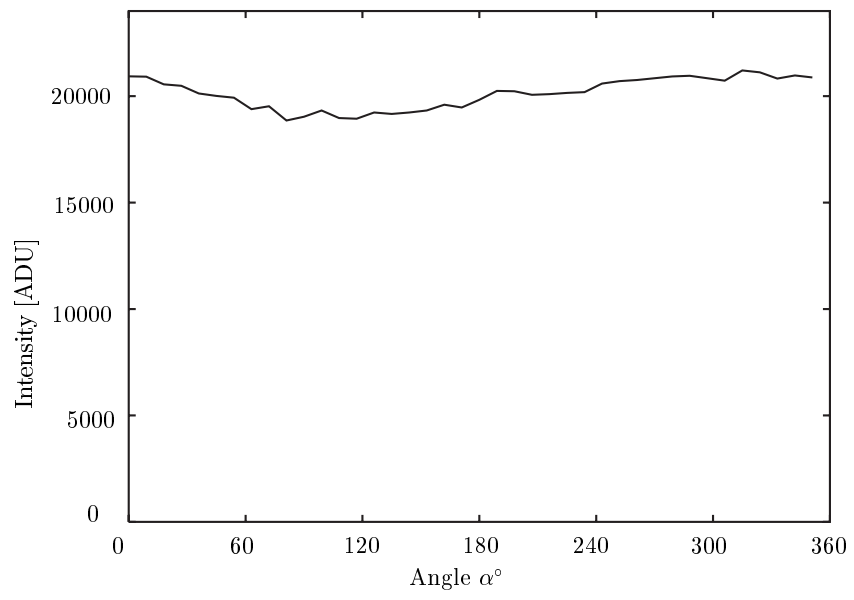

Fig. 9. An example of the intensity profile around the circular image of an unpolarized star ( $\alpha$ Leo), as in Fig. 4.

the standard errors being larger than expected from estimates based on the photon accumulations of each frame. It is unlikely that these determined values relate to the star itself but represent an instrumental polarization generated either in the fore-optics of the system, or by the modulator, or by the data reduction process. An example $I(\alpha)$ curve is given in Fig. 9 which clearly depicts a nonflat signal but one with an asymmetric variation with a minimum at around $100^{\circ}$, the dominant oscillation being the fundamental rate, $\alpha$, rather than $2 \alpha$, as expected from a source carrying a polarization. This rogue signal, most likely introduced by the poor quality rotation mechanism, gives rise to components that leak through the data reduction process, giving a "false" polarization. The fact that the $I(\alpha)$ form varies a little from one frame to the next reflects a suspected (audible) instability in the mechanism producing the rotation of the modulator, in turn giving rise to larger than expected standard errors.

This simple design of the modulator provides illumination of the CCD chip with fully polarized light, the direction of vibration rotating as the image annulus is described. With the pixel size being only $\sim 15 \times$ larger than the wavelength of the detected radiation, the possibility of the chip displaying a sensitivity according the direction of vibration, as is well known for diffraction gratings, is very important. During the cycle, the vibration turns from being parallel to the edges of the pixels to being across their diagonals. The first order effect of any such sensitivity would be produce a spurious signal with a $\cos 4 \alpha$ modulation. No evidence for this can be seen in Fig. 9 and upper limits of any polarizational effects suggest that the pixel response does not change by more than $0.1 \%$ according to the direction of polarization illuminating the chip.

The experiments clearly suggest that it would be very worthwhile to pursue this design of stellar polarimeter. The critical feature in any future instrument is the basic rotation mechanism for the modulator. It is suggested that the best design might employ a hollow shafted motor encasing the wedge prism and polarizer. Also, a design might be considered simply using a Rochon prism as the modulator. With such an optical device, one resolved component would be undeviated, providing a central point image which could act as means of determining the center of the ring and offering supplementary photometry, while the orthogonally resolved component is deviated and would provide the ring image on rotation of the prism. As this second beam exhibits mild wavelength dispersion, it might be possible to explore any coarse $p(\lambda)$ variations, all within a single CCD frame.

Acknowledgements. We are pleased to acknowledge the help and workshop skills of Colin Hunter in producing the prototype instrument from a variety of scrap material and for his help in establishing the equipment in the laboratory and on the telescope.

\section{References}

Behr, A. 1959, Veröff. der Univ.-Sternwarte zu Göttingen, 126 Clarke, D., \& Naghizadeh-Khouei, J. 1994, AJ, 108, 687

Clarke, D., \& Naghizadeh-Khouei, J. 1997, in Sensors and their Applications VIII, ed. A. T. Augousti, \& N. M. White (IOP Pubs., Bristol), 303

Magalhães, A. M., Rodrigues, C. V., Margoniner, V. E., \& Pereyra, A. 1996, in Polarimetry of the Interstellar Medium, ASP Conf. Ser. 97, ed. W. G. Roberge, \& D. C. B. Whittet, 118

Scarrott, S. M. 1991, Vistas Astron., 34, 163

Schmid, H. M., \& Schild, H. 1994, A\&A, 281, 145

Stewart, B. G. 1985, A\&A, 143, 235

Treanor, P. F. 1968, MNRAS, 138, 325 